\title{
A simplified approach to determine the ventriculoaortic junction diameter in the reimplantation procedures
}

Hiroshi Tanaka, MD, Yoshikatsu Nomura, MD, Hirohisa Murakami, MD, and Shunsuke Miyahara, MD, Hyogo, Japan

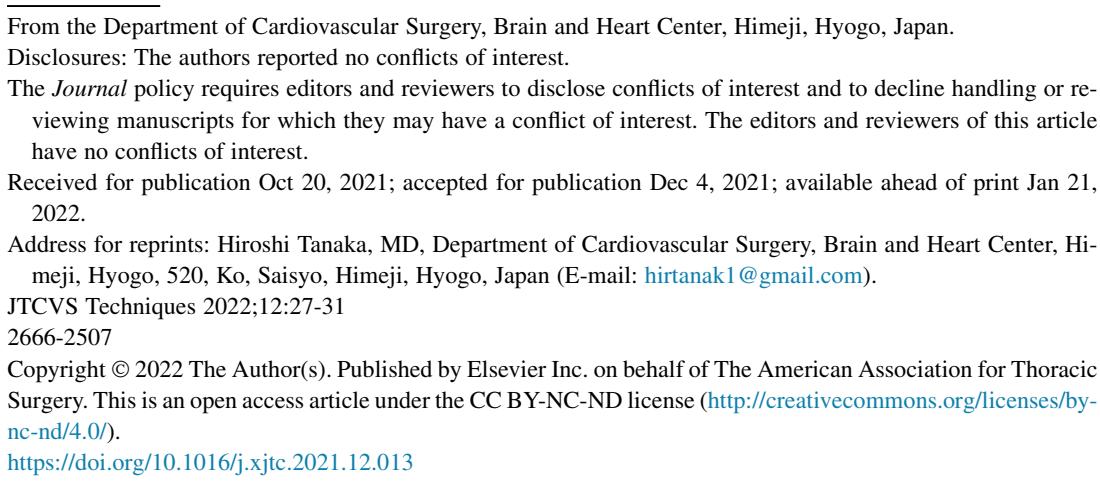

In aortic valve-sparing procedures, sufficient effective height $(\mathrm{EH})$ is essential for long-term aortic valve durability. ${ }^{1}$ The plication of the nodule of Arantius (central plication) and the free margin resuspension with polytetrafluoroethylene sutures are performed to obtain sufficient $\mathrm{EH}$, but overcorrection brings the immobility of the cusp and the gap in the central coaptation zone. In the reimplantation procedure, reducing the ventriculoaortic junction (VAJ) diameter with the proximal suture line is another option for increasing the coaptation height ${ }^{2}$; however, the degree of the reduction must be decided at the beginning of the procedure before the cusp configuration is completed. Originally, the internal radius of the VAJ was assumed to be twothirds of the cusp height and the external diameter of VAJ was obtained by adding 4 to $6 \mathrm{~mm}$ to the internal diameter, which was equal to the graft diameter to be used. ${ }^{3}$ David and colleagues $^{4}$ reported the excellent long-term outcomes of the procedure and clarified the validity of the theory. With the recent advancement of cardiac computed tomography (CT), aortic root anatomy has been well understood as performed before transcatheter aortic valve implantation. We characterized the postreimplantation root anatomy using cardiac CT and investigated the relationship among the intraoperatively measured cusp height (geometric height: $\mathrm{GH}$ ), the $\mathrm{EH}$, and the VAJ diameter.

All image acquisitions were performed using a thirdgeneration dual-source CT scanner. We used a standard protocol of electrocardiographically gated coronary arterial CT angiography. All image analyses were performed using a commercially available workstation (Ziostation2 version 2.9.7.1; AMIN Co, Ltd, Tokyo, Japan; Ziosoft Inc, Tokyo, Japan). The VAJ plane was semiautomatically defined by

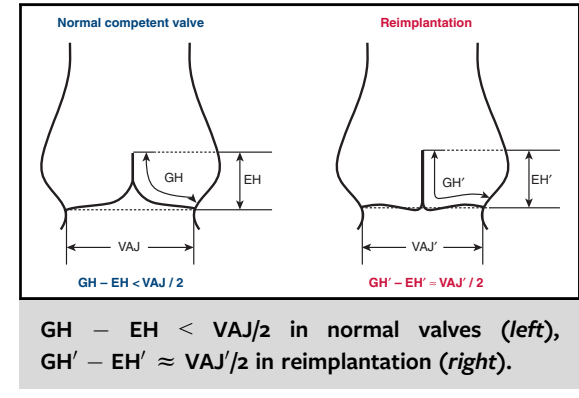

\begin{abstract}
CENTRAL MESSAGE
Ventriculoaortic junction diameter was associated with effective height and cusp height after reimplantation; therefore, the postoperative optimal veitriculoaortic junction diameter is predictable.
\end{abstract} See Commentary on page 32.

identifying the nadir of each aortic valvular hingeline (the nadir of each sinus).

A perpendicular line was given from the top of the central coaptation of cusps to the VAJ plane, the distance from the intersection of them to the nadir of each sinus was measured, and then the average length of them was taken as the radius of VAJ. The length of the perpendicular from the top of central coaptation of cusps to the VAJ plane was defined as EH. The study protocol was reviewed and approved by our institutional review board, and the need for additional informed consent was waived (Brain and Heart Center, Himeji, B210050, October 15, 2021).

Cardiac CT and the measurement of the values were performed in 27 patients (all had tricuspid aortic valve), and then we noticed that the EH after the reimplantation was approximately equal to the coaptation height (Figure 1,A). The proximal sutures in the reimplantation might cause restrictive effect for the hinge point of the cusps, which would induce such a phenomenon. Because of the difference in $\mathrm{GH}$ and $\mathrm{EH}$ of each cusp, the mean 


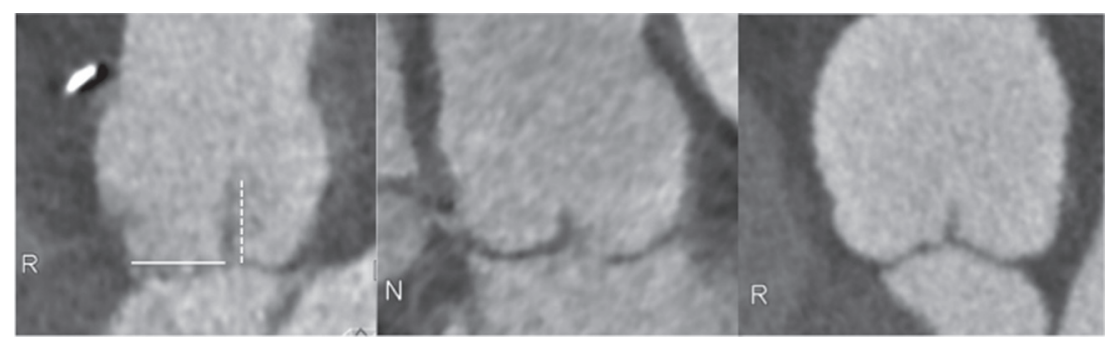

A

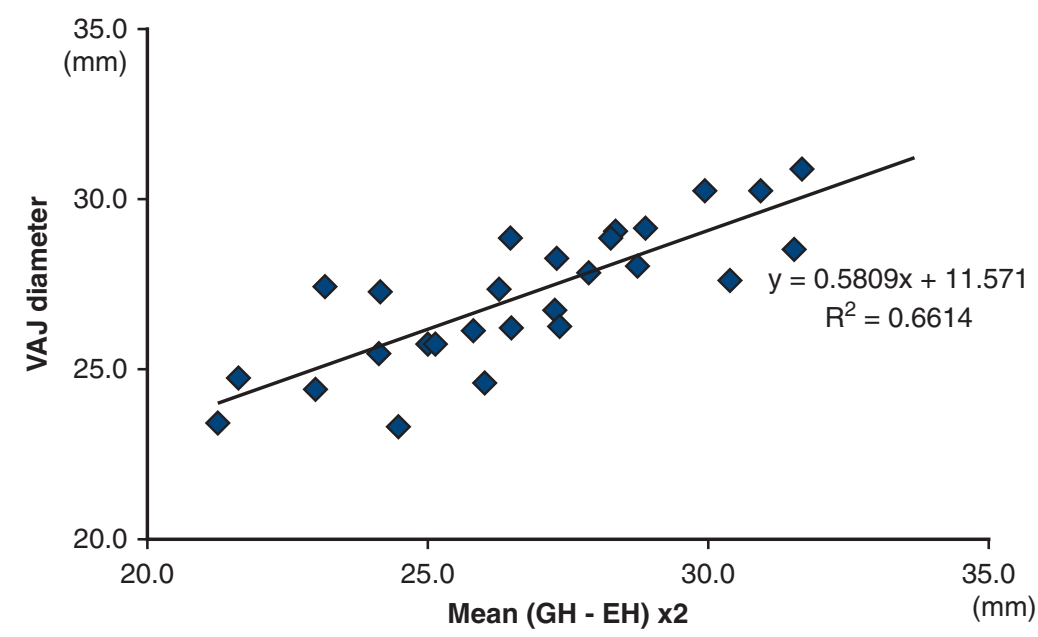

B

FIGURE 1. A, The length of the solid white line shows VAJ radius of the right coronary sinus, and the length of the dotted white line shows the EH of the right coronary cusp. Left: reimplantation, sufficient EH; center: reimplantation, low EH, and right: remodeling without annuloplasty. In the reimplantation, regardless of the relationship between GH and VAJ diameter, the coaptation length was approximately equal to the effective height. B, The black line shows the correlation between VAJ diameter and mean $(\mathrm{GH}-\mathrm{EH}) \times 2$. $R$, Right coronary cusp; $N$, non coronary cusp; VAJ, ventriculoaortic junction; $G H$, geometric height; $E H$, effective height.

value of each $(\mathrm{GH}-\mathrm{EH}) \times 2$ was adopted to compare to the VAJ diameter. There was correlation between the mean $(\mathrm{GH}-\mathrm{EH}) \times 2$ and the VAJ diameter (Figure 1, $B$, and Table E1).

\section{Normal competent valve}

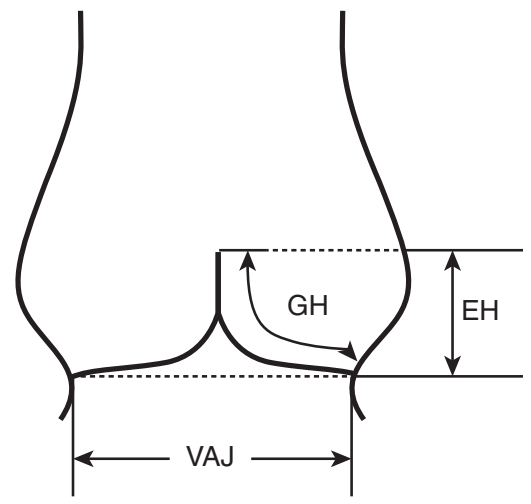

GH - EH $<$ VAJ / 2
Based on the results, the optimal VAJ diameter could be predicted by the intraoperatively measured $\mathrm{GH}$ and the target EH. Although the correlation was represented with the complex equation, the dots were included in the

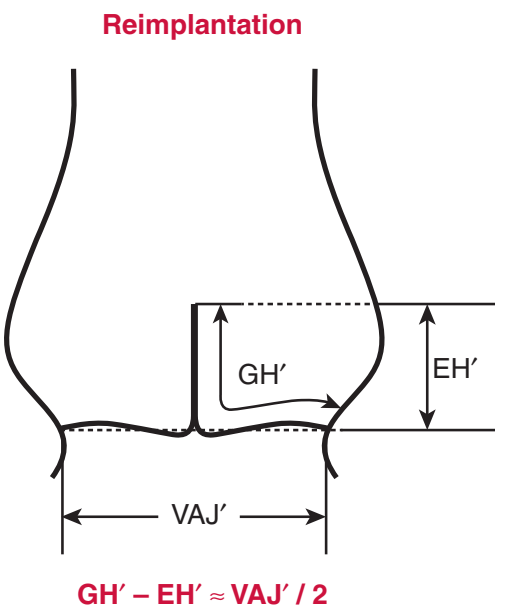

FIGURE 2. $\mathrm{GH}-\mathrm{EH}<\mathrm{VAJ} / 2$ in normal competent valve (left); however, $\mathrm{GH}^{\prime}-\mathrm{EH}^{\prime} \approx \mathrm{VAJ}^{\prime} / 2$ in the reimplantation (right). GH, Geometric height, $E H$, effective height; $V A J$, ventriculoaortic junction. 
range of the simplified equation (the mean [GH $\mathrm{EH}] \times 2 \pm 3=\mathrm{VAJ}$ diameter) (Figure 2 and Figure E1). To obtain sufficient EH even in the smallest cusp in each patient, the modified formula ([the minimum $\mathrm{GH}$ - the target $\mathrm{EH}] \times 2=$ VAJ diameter) was adopted in the latest 6 patients. Before tying down the aortic proximal sutures, we inserted a Hegar dilator of 2 to $3 \mathrm{~mm}$ smaller size than the estimated VAJ diameter into the left ventricular outflow tract, the sutures below the commissures were tied down tightly, and then the others were just taut (Figure E2). In those patients, postoperative VAJ diameter was almost identical to the estimated values, and the $\mathrm{EH}$ was above the target value $(9 \mathrm{~mm})$ in all cusps. In case of preoperative severe cusp prolapse with cusp bending, central plication was expected to be definitely necessary. Central plication might reduce cusp height; therefore, a smaller estimated VAJ diameter than that from the formula was adopted to obtain sufficient coaptation. We refer to echocardiography data from the Japanese normal population ${ }^{5}$ and set the limit value of the reduced VAJ diameter $>13 \mathrm{~mm} / \mathrm{m}^{2}$ in avoidance of aortic stenosis caused by overreduction of VAJ diameter. In the meantime, there are no patients showing mean pressure gradient through aortic valve $>15 \mathrm{~mm} \mathrm{Hg}$. In summary, a simplified formula using the intraoperatively measured $\mathrm{GH}$ and the target $\mathrm{EH}$ could predict the optimal VAJ diameter.

\section{References}

1. Schäfers HJ, Schmied W, Marom G, Aicher D. Cusp height in aortic valves. J Thorac Cardiovasc Surg. 2013;146:269-74.

2. de Kerchove L, Mastrobuoni S, Boodhwani M, Astarci P, Rubay J, Poncelet A et al. The role of annular dimension and annuloplasty in tricuspid aortic valve repair. Eur J Cardiothorac Surg. 2016;49:428-37.

3. David TE, Feindel CM. An aortic valve-sparing operation for patients with aortic incompetence and aneurysm of the ascending aorta. J Thorac Cardiovasc Surg. 1992;103:617-22.

4. David TE, David CM, Feindel CM, Manlhiot C. Reimplantation of the aortic valve at 20 years. J Thorac Cardiovasc Surg. 2017;153:232-8.

5. Daimon M, Watanabe H, Abe Y, Hirata K, Hozumi T, Ishii K, et al. Normal values of echocardiographic parameters in relation to age in a healthy Japanese population: the JAMP study. Circ J. 2008;72:1859-66. 


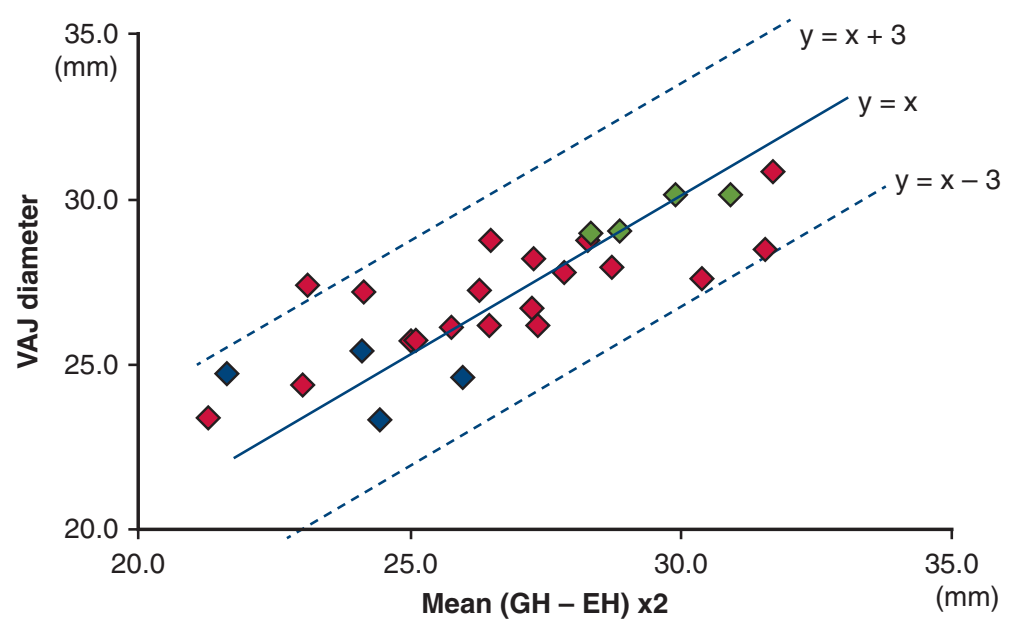

FIGURE E1. The blue solid line shows the simplified equation that VAJ diameter equals mean $(\mathrm{GH}-\mathrm{EH}) \times 2(\mathrm{y}=\mathrm{x})$, and the blue dotted line show $\mathrm{y}=\mathrm{x} \pm 3$. The graft size was selected according to the left noncommissure height (26 mm in 4; $28 \mathrm{~mm}$ in 19; and $30 \mathrm{~mm}$ in 4). Blue: $26 \mathrm{~mm}$, red: $28 \mathrm{~mm}$, and green: $30 \mathrm{~mm}$. VAJ, Ventriculoaortic junction; $G H$, geometric height, $E H$, effective height.

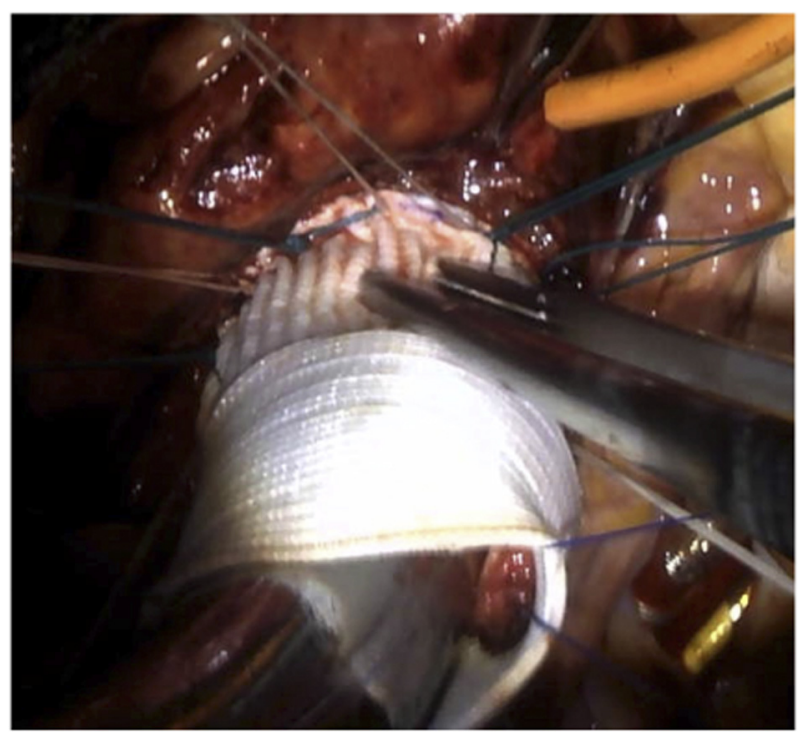

FIGURE E2. The proximal sutures are being tied with insertion of a Hegar dilator into the left ventricular outflow tract. In tricuspid aortic valve, we pull up the commissures vertically on the original position in the graft and then move the position slightly if minor prolapse exists. Major prolapse is corrected with cusp plication or free margin resuspension. The EH was $9.4 \pm 0.4 \mathrm{~mm}$ in 6 patients with the simplified formula, whereas $7.0 \pm 1.3 \mathrm{~mm}$ in the remaining 21 patients. 
TABLE E1. Measurements of cardiac computed tomography in the reimplantation

\begin{tabular}{lrrrr}
\hline & L & R & N & Mean \\
\hline $\mathrm{GH}, \mathrm{mm}$ & $20.8 \pm 2.0$ & $21.1 \pm 2.0$ & $22.4 \pm 2.3$ & $7.9 \pm 1.4$ \\
$\mathrm{EH}, \mathrm{mm}$ & $7.7 \pm 1.5$ & $7.6 \pm 1.6$ & $14.5 \pm 2.4$ & $13.7 \pm 1.7$ \\
$\mathrm{GH}-\mathrm{EH}, \mathrm{mm}$ & $13.1 \pm 2.0$ & $13.5 \pm 2.4$ & $13.4 \pm 1.8$ & $13.5 \pm 2.1$ \\
\hline Basal ring radius, $\mathrm{mm}$ & $13.2 \pm 1.6$ & $13.9 \pm 2.3$ & &
\end{tabular}

$L$, Left coronary cusp; $R$, right coronary cusp; $N$, non coronary cusp; $G H$, geometric height; $E H$, effective height. 